\title{
Sleep and circadian rhythms in hospitalized patients with decompensated cirrhosis: effect of light therapy
}

*De Rui M, **Middleton $\mathrm{B}, *$ Sticca A, *Gatta A, *Amodio $\mathrm{P}, * *$ Skene DJ, *Montagnese $\mathrm{S}$

*Department of Medicine (DIMED), University of Padova, Italy

**Faculty of Health and Medical Sciences,University of Surrey, Guildford, UK.

\section{For correspondence:}

Sara Montagnese

Dipartimento di Medicina

Via Giustiniani, 2

35128 Padova, Italia

Tel +390498218675

Fax +390498754179

sara.montagnese@unipd.it

\section{Funding:}

This work was part-funded by a grant from the Italian Ministry of Health to MS (Giovani Ricercatori 2009);

Stockgrand Ltd. (UK) undertook the urinary 6-sulphatoxymelatonin assays;

Derungs-Waldmann Illuminotecnica (Italy) provided the lighting equipment;

DJS is a Royal Society Wolfson Research Merit Award holder.

\section{Conflict of Interest:}

Prof. Debra Skene and Dr. Benita Middleton are co-directors of Stockgrand Ltd. 


\begin{abstract}
Patients with liver cirrhosis often exhibit sleep-wake abnormalities, which are, at least to some extent, circadian in origin. A relatively novel non-pharmacological approach to circadian disruption is appropriately timed bright light therapy. The aims of this pilot study were to investigate sleepwake characteristics of a well-characterized population of inpatients with cirrhosis, and to evaluate the efficacy of bright light therapy in the hospital setting. Twelve consecutive inpatients with cirrhosis underwent complete sleep-wake assessment, to include qualitative and semi-quantitative (actigraphic) indices of night-time sleep quality, daytime sleepiness, diurnal preference, habitual sleep timing, quality of life, mood and circadian rhythmicity [i.e. urine collections for measurement of the melatonin metabolite 6-sulphatoxymelatonin (aMT6s)]. Patients showed extremely impaired night sleep quality (Pittsburg Sleep Quality Index global score: 16.3 \pm 2.1 ) and daytime sleepiness was common (Epworth Sleepiness Scale: $8.3 \pm 3.2$ ). Five patients were randomly assigned to a single room in which lighting was controlled in relation to timing, spectral composition and intensity (lights on at 06:30 and off at 22:30, blue-enriched, more intense light in the morning, red-enriched, less intense light in the afternoon/evening); the others stayed in identical rooms with standard lighting. Sleep diaries revealed poor sleep quality, prolonged sleep latency $(67 \pm 138$ min) and a reduced sleep efficiency $(69 \pm 21 \%)$. These features were confirmed by actigraphy (sleep efficiency: $71 \pm 13 \%$; fragmentation index: $55 \pm 15 \%$ ). Quality of life was globally impaired, and mood

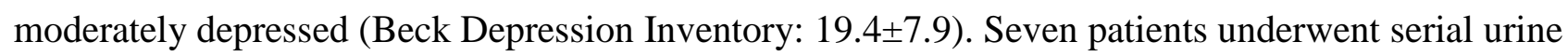
collections: no circadian aMT6s rhythm was detected in any of them, neither at baseline, nor during the course of hospitalization in either room $(n=4)$. In conclusion, sleep and circadian rhythms in hospitalized, decompensated patients with cirrhosis are extremely compromised. Treatment with bright light therapy did not show obvious, beneficial effects, most likely in relation to the severity of disturbance at baseline.
\end{abstract}

Key words: liver cirrhosis, sleep, circadian rhythms, light therapy, melatonin 


\section{Introduction}

Up to $50-65 \%$ of patients with cirrhosis of the liver complain of sleep-wake disturbances, mainly delayed sleep-wake habits, increased sleep latency and sleep fragmentation. These are considerably more common in patients with cirrhosis compared to patients with other chronic diseases, for example renal failure [1]. Less information, however, is available on the sleep-wake profile of hospitalized, very decompensated patients with cirrhosis $[2,3]$.

Sleep-wake rhythms are regulated by the interaction of two processes: the homeostatic one, which is a pressure system (i.e. the longer the wake period, the higher the likelihood of falling asleep) and a circadian one, which is regulated by the circadian timing system and light-dark cycle [4]. While limited data are available onthe homeostatic regulation in patients with cirrhosis, their circadian timing system has been studied in some depth. These patients exhibit delayed melatonin rhythms, in association with delayed sleep habits, which are probably due to a combination of impaired sensitivity to light and delayed hepatic melatonin metabolism [5-7]. This type of circadian disturbance is not unlike jet-lag East, or delayed sleep phase syndrome (DSPS), a condition characterized by sleep onset and wake times that are considerably delayed relative to conventional sleep-wake times [8]. Patients with this syndrome find it difficult to initiate sleep before 02:00 $\mathrm{h}$ and, when free of societal constraints, wake between 10:00 h and 13:00 h. Enforced, conventional wake times can result in chronically insufficient sleep, excessive day-time sleepiness and, ultimately, impaired night sleep quality [9]. As for a traveler flying East, the goal of DSPS treatment is advance of the circadian clock to entrain to the $24 \mathrm{~h}$ light-dark cycle by chronotherapy: bright light administration in the morning, with/without administration of exogenous melatonin in the evening.

Chronotherapy has been formally studied and shown to be beneficial in well-defined populations, such as shift workers [10,11], subjects with jet-lag [12] and, as previously mentioned, patients with DSPS [8]. In patients with cirrhosis, the use of evening melatonin may not be safe due to the known impairment in melatonin metabolism, which could cause accumulation of exogenous 
melatonin and high morning/daytime melatonin levels, which may worsen rather than improve these patients' condition [13]. By contrast, morning bright light administration may be a reasonable option in this patient population.

The efficacy of bright light administration relates to: $a$ ) time of administration; $b$ ) intensity; c) spectral composition and $d$ ) dynamics, i.e. how these features change across time of day. The importance of the spectral composition is due to the fact that intrinsically photosensitive retinal ganglion cells (ipRGCs), which are non rod- non cone-photoreceptors and contribute less to image formation, contain a photopigment called melanopsin. Melanopsin containing ipRGCs are maximally stimulated by light of wavelengths between 460 and $500 \mathrm{~nm}$, and are considered largely responsible for circadian entrainment $[14,15]$. Therefore, in recent years, studies have been devoted to testing the effects of blue-enriched versus standard light as a chronotherapeutic agent [16].

The aim of the present, pilot study was to investigate sleep-wake characteristics and circadian rhythms in decompensated inpatients with cirrhosis, and to assess the effect of appropriately timed blue-enriched light therapy in this population. 


\section{Patients and Methods}

Twelve consecutive, medicated inpatients with cirrhosis of the liver ( 9 males, age \pm SD: $59 \pm 15$ years) admitted to the Regional Centre for Liver Diseases of the Department of Medicine of Padova University Hospital were enrolled. Of these, 4 (all males, age \pm SD: $54 \pm 5$ years) were hospitalized for more than 10 days. Two of the 12 patients (17\%) had a urinary catheter, and $3(25 \%)$ had a diaper, and were thus unable to collect urine (vide infra); $8(67 \%)$ of the patients were on loop diuretics. Diagnosis of cirrhosis was clinical, biochemical, radiological and, where necessary, histological. Patients were classified in relation to the severity of hepatic disease, based on both the Child-Pugh [17] and MELD [18] scores. Exclusion criteria were specific, diagnosed sleep-wake disturbances (i.e. obstructive sleep apnea or restless legs syndrome), shift work, and/or treatment with psychoactive drugs or drugs known to affect sleep/melatonin metabolism. The study was conducted between October and May, to avoid the confounding effect of the sunny summer months.

\section{Night-time sleep quality}

The Pittsburg Sleep Quality Index (PSQI) evaluates subjective sleep quality during the previous month and can distinguish "good sleepers" from "poor sleepers". It is a self-administered questionnaire based on 24 questions with 4 possible answers referring to 7 domains; the latter are scored from 0 to 3 ( 0 =best). The PSQI total score is the sum of all domains, and a total score $>5$ characterizes "poor sleepers" (range: 0-21) [19].

The Sleep Timing and Sleep Quality Sleep questionnaire (STSQS) allows collection of a subjective score of night sleep quality on a scale from 1 to 9 (1=best sleep ever, $9=$ =worst sleep ever) and provides summary information on sleep-wake habits [20].

Actigraphy. An actigraph is a small device, similar to a wristwatch and worn on the non-dominant wrist, which records movement by use of an accelerometer. The simple assumption underlying the technique is wake=movement; sleep=lack of movement. Each inpatient wore an Actiwatch ${ }^{\circledR}$ (Cambridge Neurotechnology Ltd., Cambridge, UK) during the whole period of hospitalization. 
Data were then downloaded and analyzed by the software provided (Actiwatch Activity \& Sleep Analysis 5', version 5.10, Cambridge Neurotechnology Ltd., Cambridge, UK). The time period 22.30-06.30 h was referred to as night (controlled lighting system off) and used for analysis. The following indices were extracted: actual sleep time, sleep latency (i.e. time to fall asleep), sleep efficiency (i.e. percentage of time spent sleeping out of the total time spent in bed), immobile time (\%), total activity score, fragmentation index (an indicator of restlessness based on the ratio between the percentage time spent moving and the percentage immobility phases of over 1-min length) [21].

\section{Daytime sleepiness}

This was assessed both as habitual sleepiness in everyday life and instantaneous sleepiness at different times of the ongoing study.

The Epworth Sleepiness Scale (ESS) is a self-administered questionnaire in which patients are asked to assess their chance of dozing in eight different situations $(0=$ would never doze; $3=$ high chance of dozing). Excessive daytime sleepiness is defined by a total score $>11$ (range:0-24) [22]. The Karolinska Sleepiness Scale (KSS) is a self-administered scale in which patients rate their sleepiness over the previous 10 minutes on a $1-9$ scale $(1=$ very alert; $9=$ very sleepy, difficulty staying awake, fighting sleep) [23]. This was administered hourly, over the same period as the urine collections.

\section{Diurnal preference}

The Horne-Östberg (HÖ) questionnaire determines a subject's diurnal preference (definitely morning [score 70-86], moderately morning [59-69], intermediate [42-58], moderately evening [31-41] and definitely evening [16-30]) based on 19 self-administered questions [24].

\section{Usual sleep timing}


Patients completed daily Sleep Diaries during the whole length of their hospitalization. Information were collected on bed time, try to sleep time, sleep latency, number of awakenings, wake up time, get up time, number of daytime naps and their duration [25].

\section{Health-related quality of life}

The SF-36 (V1) Standard is a generic health-related quality of life questionnaire providing an eight-scale profile, as well as two summary measures; it does not include questions on sleep behavior [26]. The response to each question is scored, the eight profiles summated and the data transformed to a scale of 0 (worst) to 100 (best). Summary scores were calculated using Italian coefficients [27].

The Chronic Liver Disease Questionnaire (CLDQ) is a disease-specific health-related quality of life questionnaire providing scores for six domains of functional health and well-being, and a summary score. The response to each question is scored on a scale of 1 (all of the time) to 7 (none of the time); defined scores sets are averaged within each domain. Four questions explore sleepwake behaviour and the responses to these are included in the Fatigue or Emotional Function domains [28]. The Italian version of the questionnaire was utilized [29].

\section{Mood}

Beck Depression Inventory (BDI) is a scale that quantifies depression based on 21 statements, each of which is scored from 0 (best condition) to 3 (worst condition). The sum of such scores identifies minimal (score 0-9), slight (score 10-18), moderate (score 19-29) or severe (score 30-63) depression [30].

\section{6-sulphatoxy-melatonin (aMT6s) rhythm}

Sequential urine samples were collected from patients over the first two nights after inpatient admission, and the day in between (4-hour intervals during the day and 8-hour intervals overnight, 
for a total of 36 hours) [31]; $3 \mathrm{ml}$ aliquots were collected from each sample and kept at $-20^{\circ} \mathrm{C}$ until assayed for 6-sulpahtoxymelatonin (aMT6s), in duplicate, using a commercial radioimmunoassay [32]. Urine collections were repeated every 7 days, depending on the length of hospitalization. All samples for a single individual were measured in the same assay. The aMT6s profiles were evaluated using cosinor analysis, which is based on the least square approximation of the time series using a cosine function with a period of 24 hours [33]. The following parameters were obtained: 24h output: aMT6s excretion over 24 hours; acrophase time: time of the peak aMT6s concentration, or maximum of the fitted cosinor function; mesor: mean aMT6s value for all the samples included in the cosinor analysis, and amplitude: difference between the mesor and the peak aMT6s concentrations. The acrophase time was classified as normal if it occurred between midnight and 06:00 [34]. The 'goodness of fit' used to determine the validity of the cosinor-derived indices was the likelihood of the data points fitting a straight line as opposed to a cosine curve, expressed as a $\mathrm{p}$ value; data were considered acceptable if the cosinor fit was significant at the $95 \%$ level $(\mathrm{p}<0.05)$ [33].

\section{Light therapy}

Four adjacent single rooms were selected; all had the same dimensions and were situated on the same side of the ward corridor, facing North-North/West, on the ground floor of the hospital. In one of these, the standard wall-mounted lighting system switched on/off by the patient and/or personnel was replaced by a controlled lighting system (Amadea wall-mounted lamp, Derungs-Waldmann Illuminotecnica, San Giuliano Milanese, Italy). Two ballasts (39W) emitted light with varying spectrum (from $6500 \mathrm{~K}$ to $3000 \mathrm{~K}$ ) and intensity. In order to advance the sleep-wake/circadian cycle of patients with cirrhosis, the lamp was set to automatically switch on at 06:30 h and off at 22:30 h. In addition, the lighting changed dynamically across the day, being more intense and blue-enriched in the morning $(6500 \mathrm{~K})$, and becoming less intense and red-enriched during the afternoon/evening hours (turning from $6500 \mathrm{~K}$ to $3000 \mathrm{~K}$ ). The maximum intensity in the gaze direction was 500 lux. 
The patient/personnel could not operate the system; however, the room was equipped with an additional switch that would allow immediate and full lighting of the room in case of a medical emergency. 


\section{Results}

Reasons for hospitalization were cirrhosis-related in $10(83 \%)$ of the 12 patients: tense ascites $(n=3)$, hepatic encephalopathy (3), hepato-renal syndrome (3), spontaneous bacterial peritonitis (1). One patient was admitted for cholecystitis and one for pneumonia. In relation to the degree of hepatic decompensation, 1 patient was classed as Child A, 1 as B, and 10 as C; average MELD score was $21 \pm 7$. Five patients $(42 \%)$ were randomized to the room with the controlled lighting system and the remaining seven patients to standard lighting rooms. Average inpatient stay was $8.4 \pm 6.1$ days.

Eleven patients $(92 \%)$ completed the STSQS and sleep diaries, $8(67 \%)$ the ESS, BDI and SF-36, $6(50 \%)$ the PSQI and HÖ, 5 (42\%) the CLDQ. The remaining sets of questionnaires were not completed due to the severity of the condition and associated fatigue/tiredness. Hourly KSS compliance was even lower, with only 3 patients (25\%) completing the scale but not on an hourly basis as required.

No substantial differences were observed between eases and controls the intervention and control group patients in relation to age, hepatic decompensation, night-time sleep quality, daytime sleepiness, diurnal preference, quality of life and mood (Table 1). Sleep timing and actigraphy were also comparable (Table 1). Patients showed profoundly impaired sleep quality (PSQI global score: 16.3 \pm 2.1 , STSQS sleep quality: $6.3 \pm 2.5)$. They all had intermediate (50\%) or moderately morning (50\%) diurnal preference. Two (18\%) had excessive daytime sleepiness (abnormal ESS). Sleep diaries were consistent with subjectively reported sleep disturbances, documenting prolonged sleep latency $(67 \pm 138 \mathrm{~min})$ and reduced sleep efficiency $(69 \pm 21 \%)$. Sleep onset occurred at $23.7 \pm 2.2 \mathrm{~h}$ and wake up at $6.3 \pm 1.6 \mathrm{~h}$ with $3.4 \pm 1.8$ awakenings per night. Actigraphic data also confirmed

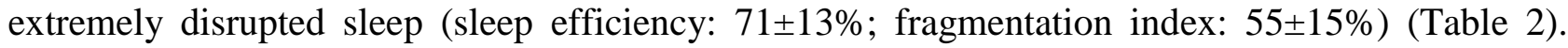
Quality of life was globally impaired and mood was moderately depressed (BDI: 19.4 \pm 7.9 )(Table $1)$.

Baseline sequential $36 \mathrm{~h}$ urine collections were possible in 7 (3 in the room with controlled 
lighting system) out of the 12 patients. Four of these underwent a second sequential $36 \mathrm{~h}$ urine collection at 7 days from admission, and two a further one, at 14 days (Figure 1). No significant cosine fit was detected in any of the aMT6s profiles (Figure 1), thus cosinor analysis parameters are not presented. At baseline, average aMT6s output was $12.2 \pm 7.9 \mu \mathrm{g} / 24 \mathrm{~h}$, and was comparable in eases the intervention and control group $(13.8 \pm 16.9 \mu \mathrm{g} / 24 \mathrm{~h} v s .10 .6 \pm 5.7 \mu \mathrm{g} / 24 \mathrm{~h}, \mathrm{p}>0.05)$. No significant correlations were observed between aMT6s 24h output and serum creatinine levels.

No significant differences were observed between the intervention and control rooms in the evolution of the patients' sleep-wake profiles (diaries and actigraphy) over the length of hospitalization. However, wake-up times were earlier in patients admitted into the treatment room (Table 2). Bright, dynamic blue-enriched lighting had no obvious effects on the cosine fit of the aMT6s profiles (Figure 1), which remained not significant. However, visual analysis of the repeated aMT6s profiles suggested phase advance in two patients, who were both admitted into the controlled lighting system room (Figures 1D and 1G). 


\section{Discussion}

Patients enrolled in the present study were severely decompensated, and exhibited profoundly impaired sleep-wake and circadian melatonin rhythms. Bright daytime blue-enriched light treatment did not have any obvious beneficial effects, most likely in relation to the severity of sleep/circadian disturbance at baseline. However, visual analysis of the sleep-wake profiles and the urinary aMT6s of the patients treated with light suggested some degree of phase advance, still within the context of aMT6s arrhythmia.

In the group studied here, quality of life was poor, as previously observed by other authors [35-37], and possibly even worse, particularly in relation to the "physical" and "emotional role" domains, as well as the global health one, global physical health being worse than global mental health. These data are in agreement with previously published data from our own and from other authors, suggesting that the physical domains of quality of life are more affected than the mental domains in these patients $[35,36]$. Mood was depressed, also in agreement with previously published work [38].

Patient compliance with the study procedures was poor, especially regarding actigraphy and to the repeated questionnaires, most likely due to their severely compromised clinical and psychological status. As previously observed, compliance was slightly better with simple, short questionnaires compared to the more complex ones [20]. Sleep questionnaires, sleep diaries and actigraphy all showed profoundly altered night-time sleep quality, with very prolonged sleep latency and extremely fragmented night sleep. All patients had morning/intermediate diurnal preference, thus the previously reported association between impaired sleep quality and eveningness in cirrhosis could not be studied in this group $[1,35]$.

No significant cosine fit for the aMT6s rhythm was detected in any of the patients. We have previously reported [13] no detectable rhythm in 57\% of decompensated outpatients with cirrhosis. The findings in the present study document $100 \%$ lack of rhythm. This could be a result of decompensation, medication and/or hospitalization. Urinary aMT6s 24h output, however, was 
similar to that described previously [13] and more recently by Chojnacki et al. [39], confirming desynchronization of melatonin/aMT6s timing rather than abnormal melatonin production in this patient population. These findings could relate to both decompensation of cirrhosis, as previously suggested [40], and also to the hospital environment, in which patients are often disturbed across the 24-hours in relation to drug and food delivery, and management of other patients who are in nearby rooms. In addition, there is a possibility that an abnormally late melatonin/aMT6s peak, which can be detected in decompensated patients with cirrhosis in very tightly controlled light conditions [5,6] might have been suppressed by morning light, thus completely masking the underlying rhythm. Furthermore, kidney failure may have impinged on urinary aMT6s excretion, although the profiles of the two patients with hepato-renal syndrome did not seem different from the remaining five. In addition, a significant proportion of the patients were on diuretics. To our knowledge, no literature data are available on the effect of diuretics on the excretion of aMT6s or other melatonin metabolites. While we have not tested this directly in our study, the fact that aMT6s excretion was calculated as ngr per hour (as opposed to ngr per volume unit) should contain diuretics-related bias, if any. In addition no significant relationships were observed between daily aMT6s output and serum creatinine. Finally, since aMT6s is a small molecule, it should be freely excreted and probably not reabsorbed; this suggest that the influence of diuretics, and especially loop diuretics, should be limited.

In this setting, the lack of an obvious effect of light administration is reasonable, as light can strengthen or shift an existing rhythm, but cannot restore an absent rhythm, which was the baseline situation for all the patients enrolled. However, visual analysis of the repeated aMT6s profiles suggested some degree of phase advance in two patients, both in the controlled lighting system room. We have previously documented a beneficial effect of bright light therapy in a patient with compensated cirrhosis of the liver and sleep-wake inversion [41]. Although aMT6s was not measured in this subject, she appeared to have some degree of sleep-wake rhythmicity, albeit abnormal. Other authors have utilized bright light administration in the hospital setting and have 
succeeded in increasing total sleep time and in decreasing night awakenings [42, 43]. In these studies, however, light intensity was higher, and the patient population was not as compromised from a medical standpoint. Riemersma-van der Lek et al. [44] found that light therapy and melatonin treatment in demented people ameliorated sleep architecture nocturnal restlessness, sleep efficiency and sleep fragmentation. We chose not to administer melatonin in addition to light, as we were concerned about a possible negative effect due to reduced/delayed hepatic metabolism [13].

The limitations of this study are several, including the small number of patients recruited, their only partial compliance with study procedures, and the use of actigraphy in substantially bedridden individuals. However, these pilot data seem useful as: $i$ ) they help to highlight the difficulties of studying sleep-wake/circadian rhythms in a clinical setting [20,45], and the need for more patient-friendly tools and study protocols; ii) they suggest that further, formal studies on the effects of chronotherapy in less decompensated outpatients with cirrhosis may be worthy. Finally, as there is limited interest for circadian rhythms and light hygiene in routine hospital medicine, the concept of adaptable lighting in the hospital setting is in itself an innovation, and may attract the attention of both doctors and health administrators. 


\section{Figure legends}

Figure 1. Individual urinary aMT6s profiles over $36 \mathrm{~h}$ collections at baseline and, where available, during the hospitalization period (7 and 14 days [d 7/14]) in consecutive patients, as enrolled. Each point represents the middle time of the collection period. Each patient is briefly characterized in relation to origin/degree of decompensation of their cirrhosis, reason for hospital admission and room type. The levels of urinary aMT6s were extremely variable (y axis scales vary from panel to panel and some of the apparent, magnified peaks/oscillations may have limited clinical significance). No significant cosine fit for the aMT6s rhythm could be detected in any patient at baseline, or during hospitalization, in either room type. However, two patients showed some degree of phase advance on visual analysis [panel D: the nadir of an extremely delayed and weak rhythm seems to shift backwards from 20:00 to 16:00 hr (arrow) on the day 7 compared to baseline collection; panel G: aMT6s levels increase in a more physiological fashion during the night (arrow) on the day 7 compared to baseline collection]; both patients were admitted in the controlled lighting system room. 


\section{References}

1. Cordoba J, Cabrera J, Lataif L, Penev P, Zee P, Blei AT. High prevalence of sleep disturbance in cirrhosis. Hepatology 1998;27(2):339-345.

2. Hourmand-Ollivier I, Piquet MA, Toudic JP, Denise P, Dao T. Actigraphy: A new diagnostic tool for hepatic encephalopathy. World J Gastroenterol 2006;12(14):2243-2244.

3. Cordoba J, Steindl P, Blei AT. Melatonin arrhythmia is corrected after liver transplantation. Am J Gastroenterol 2009;104(7):1862-1863. doi: 10.1038/ajg.2009.171.

4. Borbely AA. A two process model of sleep regulation. Hum Neurobiol 1982;1(3):195-204.

5. Steindl PE, Finn B, Bendok B, Rothke S, Zee PC, Blei AT. Disruption of the diurnal rhythm of plasma melatonin in cirrhosis. Ann Intern Med. 1995;123(4):274-7.

6. Montagnese S, Middleton B, Mani AR, Skene DJ, Morgan MY. On the origin and the consequences of circadian abnormalities in patients with cirrhosis. Am J Gastroenterol 2010;105(8):1773-1781. doi: 10.1038/ajg.2010.86.

7. Montagnese S, De Pittà C, De Rui M, Corrias M, Turco M, Merkel C, Amodio P, Costa R, Skene DJ, Gatta A. Sleep-wake abnormalities in patients with cirrhosis. Hepatology 2014;59(2):705-712. doi: 10.1002/hep.26555.

8. Weitzman ED, Czeisler CA, Coleman RM, Spielman AJ, Zimmerman JC, Dement W, Richardson G, Pollack CP. Delayed sleep phase syndrome. A chronobiological disorder with sleep-onset insomnia. Arch Gen Psychiatry 1981;38(7):737-746.

9. Thorpy MJ, Korman E, Spielman AJ, Glovinsky PB. Delayed sleep phase syndrome in adolescents. J Adolesc Health Care 1988;9(1):22-27.

10. Ross JK, Arendt J, Horne J, Haston W. Night-shift work in Antarctica: sleep characteristics and bright light treatment. PhysiolBehav 1995;57(6):1169-1174.

11. Czeisler CA, Johnson MP, Duffy JF, Brown EN, Ronda JM, Kronauer RE. Exposure to bright light and darkness to treat physiologic maladaptation to night work. N Engl J Med 1990;322(18):1253-1259.

12. Arendt J. Managing jet lag: Some of the problems and possible new solutions. Sleep Med Rev 2009;13(4):249-256. doi: 10.1016/j.smrv.2008.07.011.

13. Montagnese S, Middleton B, Mani AR, Skene DJ, Morgan MY. Sleep and circadian abnormalities in patients with cirrhosis: features of delayed sleep phase syndrome? Metab Brain Dis 2009;24(3):427-439. doi: 10.1007/s11011-009-9146-5.

14. Lucas RJ, Peirson SN, Berson DM, Brown TM, Cooper HM, Czeisler CA, Figueiro MG, Gamlin PD, Lockley SW, O'Hagan JB, Price LL, Provencio I, Skene DJ, Brainard GC. 
Measuring and using light in the melanopsin age. Trends Neurosci. 2014;37(1):1-9. doi: 10.1016/j.tins.2013.10.004.

15. Revell VL, Arendt J, Terman M, Skene DJ. Short-wavelength sensitivity of the human circadian system to phase-advancing light. J Biol Rhythms 2005;20(3):270-272.

16. Francis G, Bishop L, Luke C, Middleton B, Williams P, Arendt J. Sleep during the Antarctic winter: preliminary observations on changing the spectral composition of artificial light. $\mathbf{J}$ Sleep Res. 2008;17(3):354-60. doi: 10.1111/j.1365-2869.2008.00664.x.

17. Pugh RN, Murray-Lyon IM, Dawson JL, Pietroni MC, Williams R. Transection of the oesophagus for bleeding oesophageal varices. Br J Surg 1973;60(8):646-9.

18. Kamath PS, Wiesner RH, Malinchoc M, Kremers W, Therneau TM, Kosberg CL, D'Amico G, Dickson ER, Kim WR. A model to predict survival in patients with end-stage liver disease. Hepatology 2001;33(2):464-70.

19. Buysse DJ, Reynolds CF, III, Monk TH, Berman SR, Kupfer DJ. The Pittsburgh Sleep Quality Index: a new instrument for psychiatric practice and research. Psychiatry Res 1989;28(2):193-213.

20. Montagnese S, Middleton B, Skene DJ, Morgan MY. Sleep-wake patterns in patients with cirrhosis: all you need to know on a single sheet. A simple sleep questionnaire for clinical use. J Hepatol 2009;51(4):690-695. doi: 10.1016/j.jhep.2009.06.006.

21. Arendt J, Middleton B, Williams P, Francis G, Luke C. Sleep and circadian phase in a ship's crew. J Biol Rhythms 2006;21(3):214-221.

22. Johns MW. A new method for measuring daytime sleepiness: the Epworth sleepiness scale. Sleep 1991;14(6):540-545.

23. Akerstedt T, Gillberg M. Subjective and objective sleepiness in the active individual. Int J Neurosci 1990;52(1-2):29-37.

24. Horne JA, Ostberg O. A self-assessment questionnaire to determine morningnesseveningness in human circadian rhythms. Int J Chronobiol 1976;4(2):97-110.

25. Lockley SW, Skene DJ, Arendt J. Comparison between subjective and actigraphic measurement of sleep and sleep rhythms. J Sleep Res 1999;8(3):175-183.

26. Ware JE, Jr., Sherbourne CD. The MOS 36-item short-form health survey (SF-36). I. Conceptual framework and item selection. Med Care 1992;30(6):473-483.

27. Apolone G, Mosconi P, Ware JE, Jr. Questionario sullo stato di salute SF-36: manuale d'uso e guida all'interpretazione dei risultati Guerini e associati; 1997. 
28. Younossi ZM, Guyatt G, Kiwi M, Boparai N, King D. Development of a disease specific questionnaire to measure health related quality of life in patients with chronic liver disease. Gut 1999;45(2):295-300.

29. Rucci P, Taliani G, Cirrincione L, Alberti A, Bartolozzi D, Caporaso N, et al. Validity and reliability of the Italian version of the Chronic Liver Disease Questionnaire (CLDQ-I) for the assessment of health-related quality of life. Dig Liver Dis 2005;37(11):850-860.

30. Beck AT, Ward CH, Mendelson M, Mock J, Erbaugh J. An inventory for measuring depression. Arch Gen Psychiatry 1961;4:561-571.

31. Lockley SW, Skene DJ, James K, Thapan K, Wright J, Arendt J. Melatonin administration can entrain the free-running circadian system of blind subjects. $\mathrm{J}$ Endocrinol 2000;164(1):R1-R6.

32. Aldhous ME, Arendt J. Radioimmunoassay for 6-sulphatoxymelatonin in urine using an iodinated tracer. Ann Clin Biochem 1988;25( Pt 3):298-303.

33. Minors DS, Waterhouse JM. Mathematical and statistical analysis of circadian rhythms. Psychoneuroendocrinology 1988;13(6):443-464.

34. Lockley SW, Skene DJ, Tabandeh H, Bird AC, Defrance R, Arendt J. Relationship between napping and melatonin in the blind. J Biol Rhythms 1997;12(1):16-25.

35. Montagnese S, Middleton B, Skene DJ, Morgan MY. Night-time sleep disturbance does not correlate with neuropsychiatric impairment in patients with cirrhosis. Liver Int 2009;29(9):1372-1382. doi: 10.1111/j.1478-3231.2009.02089.x.

36. Marchesini G, Bianchi G, Amodio P, Salerno F, Merli M, Panella C, et al. Factors associated with poor health-related quality of life of patients with cirrhosis. Gastroenterology 2001;120(1):170-178.

37. Samanta J, Dhiman RK, Khatri A, Thumburu KK, Grover S, Duseja A, et al. Correlation between degree and quality of sleep disturbance and the level of neuropsychiatric impairment in patients with liver cirrhosis. Metab Brain Dis 2013;28(2):249-259. doi: 10.1007/s11011-013-9393-3.

38. Bianchi G, Marchesini G, Nicolino F, Graziani R, Sgarbi D, Loguercio C, et al. Psychological status and depression in patients with liver cirrhosis. Dig Liver Dis 2005;37(8):593-600.

39. Chojnacki C, Walecka-Kapica E, Klupinska G, Wachowska-Kelly P, Zylinska K, Winczyk $\mathrm{K}$, et al. Serotonin and melatonin secretion and metabolism in patients with liver cirrhosis. Pol Arch Med Wewn 2012;122(9):392-397. 
40. Montagnese S, Middleton B, Mani AR, Skene DJ, Morgan MY. Changes in the 24-h plasma cortisol rhythm in patients with cirrhosis. J Hepatol 2011;54(3):588-590. doi: 10.1016/j.jhep.2010.08.015.

41. De Rui M, Gaiani S, Middleton B, Skene DJ, Schiff S, Gatta A, Merkel C, Amodio P, Montagnese S. Bright times for patients with cirrhosis and delayed sleep habits: a case report on the beneficial effect of light therapy. Am J Gastroenterol 2011;106(11):2048-2049. doi: 10.1038/ajg.2011.239.

42. Wakamura T, Tokura H. Influence of bright light during daytime on sleep parameters in hospitalized elderly patients. J Physiol Anthropol Appl Human Sci 2001;20(6):345-351.

43. Yamadera H, Ito T, Suzuki H, Asayama K, Ito R, Endo S. Effects of bright light on cognitive and sleep-wake (circadian) rhythm disturbances in Alzheimer-type dementia. Psychiatry Clin Neurosci 2000;54(3):352-353.

44. Riemersma-van der Lek RF, Swaab DF, Twisk J, Hol EM, Hoogendijk WJ, Van Someren EJ. Effect of bright light and melatonin on cognitive and noncognitive function in elderly residents of group care facilities: a randomized controlled trial. JAMA 2008;299(22):26422655. doi: 10.1001/jama.299.22.2642.

45. Gogenur I, Ocak U, Altunpinar O, Middleton B, Skene DJ, Rosenberg J. Disturbances in melatonin, cortisol and core body temperature rhythms after major surgery. World J Surg 2007;31(2):290-298. 